\title{
A 3-D Laser Scanning System and Scan Data Processing Method for the Monitoring of Tunnel Deformations
}

\author{
K. Chmelina ${ }^{\text {a, } * \text {, J. Jansa }}{ }^{\mathrm{b}}$, G. Hesina ${ }^{\mathrm{c}}$, C. Traxler ${ }^{\mathrm{c}}$ \\ ${ }^{a}$ Geodata Group, Hütteldorferstrasse 85, 1150 Vienna, Austria - klaus.chmelina@ geodata.at \\ b Inst. of Photogrammetry and Remote Sensing, Vienna University of Technology, Gusshausstrasse 27-29, 1040 Vienna, \\ Austria - jj@ipf.tuwien.ac.at \\ ${ }^{\mathrm{c}}$ VRVIS - Center for Virtual Reality and Visualisation Research, Donau-City-Strasse 1, 1220 Vienna, Austria - (hesina, \\ traxler)@vrvis.at
}

KEY WORDS: 3-d laserscanning, mobile multi sensor system, tunnel deformation monitoring, Virtual Reality

\begin{abstract}
:
The paper presents the mobile multi sensor system Orthos Plus for the monitoring and mapping of tunnel walls, a scan data processing method for the evaluation of 3-d tunnel wall displacements from subsequent wall scans and, finally, a Virtual Reality tool supporting the interpretation of data. The measuring system consists of a 3-d laserscanner, a motorized totalstation and a digital camera that are integrated on a light metal frame that is installed on a mobile platform. It has been designed to perform tunnel measurements most efficiently and to meet the special requirements of tunnels under construction. The evaluation of 3-d displacements is based on a 3-d matching algorithm that takes advantage from the particular conditions of tunnel (shotcrete) surfaces. The Virtual Reality tool allows for the viewing of data in a 3-d Virtual Reality tunnel model and of their animation in time and space in order to support their understanding in an optimal way. The measuring system Orthos Plus has been developed in the course of a national research project, the 3-d matching method in the frame of the Austrian Christian Doppler Laboratory Spatial Data from Laser Scanning and Remote Sensing and the VR tool in the Austrian COMET K1 Competence Center VRVis Center.
\end{abstract}

\section{INTRODUCTION}

During tunnel excavation the monitoring and mapping of tunnel surfaces (tunnel wall, tunnel face) is a routine (daily) procedure supporting a variety of relevant tasks such as:

- check of tunnel profile geometry (e.g. for locating underbreak/overbreak areas, for proving profile clearness)

- check of tunnel surface physical conditions (e.g. for proving the flatness of shotcrete layers prior to inner lining installation, discovery of cracks, wet areas etc.)

- calculation of quantities (e.g. volumes and costs for excavated material, shotcrete and inner lining concrete)

- tunnel support documentation (e.g. for proving the number and location of installed support means such as anchors and rock bolts)

- design and optimisation of tunnel support

- geological modelling (e.g. for updating the geological 3 -d ground model and predicting geological features such as joints)

- geotechnical interpretation (e.g. for assessing geotechnical risks, for checking the efficiency of the installed tunnel support means, for analysing and predicting 3-d tunnel deformations and system behaviour)

For acquiring the needed data, for about 30 years now, totalstations and cameras are used. Uncountable tunnel site surveyors operate them successfully day by day, worldwide. About 10 years ago laserscanning was introduced as a brand new measuring technique in tunnelling which, since then, quickly developed further. For the first four tasks listed above, the technique already became common practice in tunnelling.
On modern tunnel sites today 3-d laserscanners are part of the standard measuring equipment.

However, a suitable laserscanning system and an appropriate scan data processing method that would also support the last task of the list and allow for the determination of 3-d tunnel wall displacements are not available yet. Mainly this is due to several fundamental disadvantages of the laserscanning method compared to the well established optical 3-d displacement monitoring method that uses totalstations and prism targets:

1. As a sufficient angle of incidence is needed to obtain reliable results, a laserscanner can only measure up to about $20 \mathrm{~m}$ of tunnel length from one station (depending on the profile size) while a totalstation can measure all prism targets within a section of up to $100 \mathrm{~m}$ of length easily. Consequently, at least five times more stations and a much longer time are needed for measuring the same tunnel length with a laserscanner.

2. A laserscanner needs to be set up right in the tunnel volume to be measured (preferably in the tunnel center) while a totalstation can measure comfortably from a safe distance from behind (free-stationed flexibly wherever suitable). Consequently, laserscanning disturbs construction works much more, is not that flexible and must be carried out right in the critical (dangerous) deformation area (e.g. at the tunnel face).

3. For processing 3-d displacements from a repeatedly laserscanned surface it may not be changed between the scans. So in case another shotcrete layer is installed (which is often the case in tunnelling) the initially scanned surface simply does not exist anymore and subsequent scans cannot be related to

\footnotetext{
* Corresponding author. This is useful to know for communication with the appropriate person in cases with more than one author.
} 
previous ones. As totalstations measure to prism targets that are fix-installed on convergence bolts in boreholes, point identity is always guaranteed no matter what happens to the tunnel surface around the targets.

4. As absolute displacements must be delivered within a local project coordinate system, the georeferencing problem must be solved. This means that a laserscanner either needs to take additional measurements to reference targets, itself must be measured from extern by a totalstation or other methods must be applied. No matter what method is used, each one causes additional effort and time. With totalstations georeferencing is solved by free stationing that is highly automated and a quick procedure.

On the other hand laserscanning would have considerable advantages:

1. The huge number of prism targets would not be needed anymore which would save equipment, installation and maintenance costs and time enormously; tunnelling would become more economical.

2. It could be provided real tunnel surface displacements instead of mere target movements. This would make the often occurring question obsolete whether the observed target movements really constitute deformations. Often targets are displaced by other reasons than deformation which can lead to misinterpretation. Sometimes targets are fully destroyed and must be replaced which leaves a gap in the time series of displacements.

3. When scanning in high resolution the displacements could be determined at almost every point on the tunnel surface instead of only at a small number of fix points. This would increase the density and quantity of information tremendously and improve the quality of geotechnical interpretation. Deformations no longer would have to be interpolated between targets.

Motivated by the above promising advantages, the growing demands of geotechnical experts, the lasting trend towards full tunnel documentation and the increased capabilities of the available laserscanning soft- and hardware products an R\&D project aiming at developing the Orthos Plus system was started.

\section{MOBILE MULTI SENSOR SYSTEM ORTHOS PLUS}

\subsection{System objective}

The objective was to design a system able to most-efficiently (rapidly, automatically) acquire and georeference tunnel wall scans and images in a static (stop and go) measuring mode. Depending on the type of ground surface it should be attachable to different mobile platforms (running on rails or wheels) and operable by one single user. The system should be applicable in tunnels under construction as well as in ready-made tunnels. It should be modular, flexible and extensible and able to include sensors from different manufacturers. In addition, the acquired data should provide sufficient quality (especially resolution and accuracy) so that the system can also be used for deformation monitoring purposes.

\subsection{System design}

The current Orthos Plus prototype (figures 1 and 2) consists of a 3-d laserscanner (Riegl and Faro scanners are currently supported), a digital camera (e.g. a Nikon D90) and a robotic totalstation (e.g. Leica TPS1200 series). The three sensors are installed on a light metal frame that can be attached to a mobile platform (e.g. a hand driven trolley). The platform carries all further needed components, most basically the power supply unit and the control computer with WLAN display.

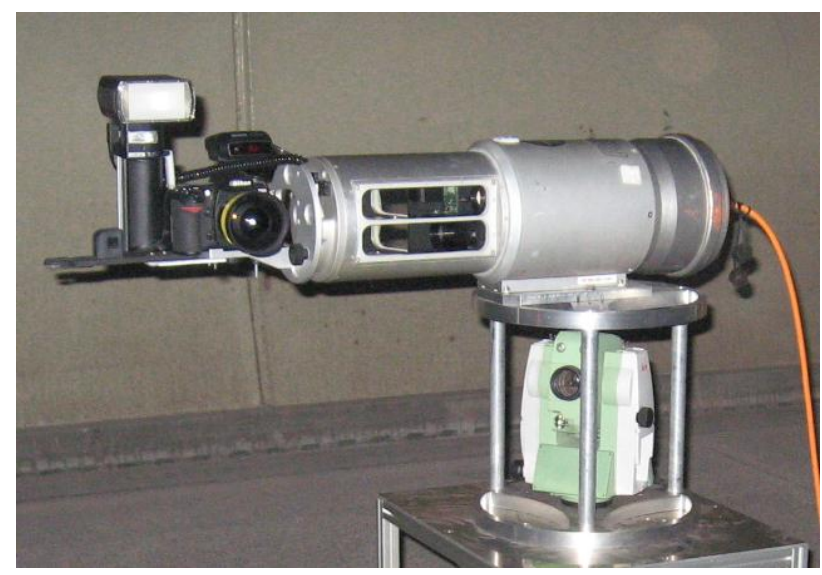

Figure 1. 3-d laserscanner (RIEGL), digital camera (NIKON) and totalstation (LEICA) on light metal frame

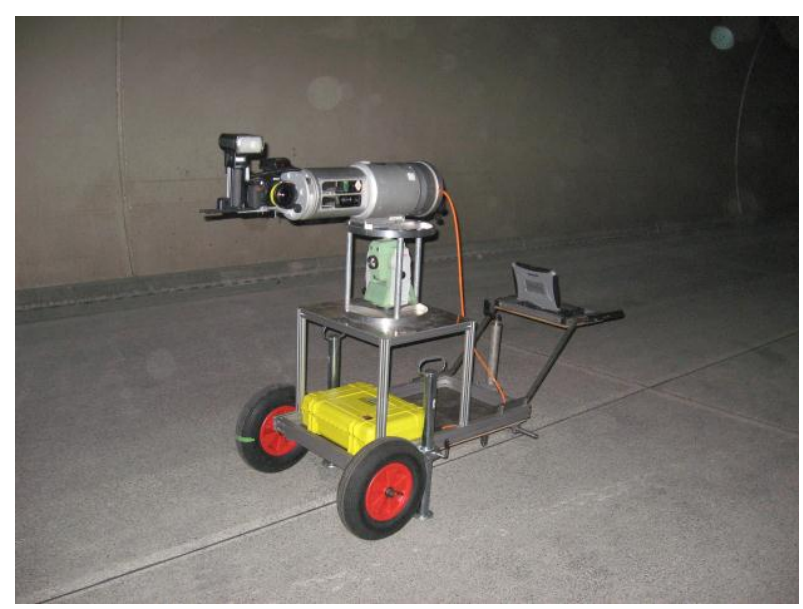

Figure 2. Light metal frame with sensors attached to trolley carrying control computer, WLAN display and power supply

The system can be remote-controlled via a WLAN touchscreen display connected to a tunnel-proof control computer on the trolley. The onboard software controls all sensors and measurements and offers special functions to quickly check the data quality already in the tunnel. Particularly, this includes the achieved georeferencing quality after applying the sevenparameter Helmert transformation.

To speed up work (to disturb construction activities as short as possible), all sensors measure in parallel, levelling of the system is disregarded and all relevant support functions such as power search, automatic target recognition, point identification and point number assignment are utilised. 
For a first and immediate check of results the calculation and display of georeferencing results and 2-d tunnel bands showing the obtained wall scan data and wall images is possible (Fig. 3).

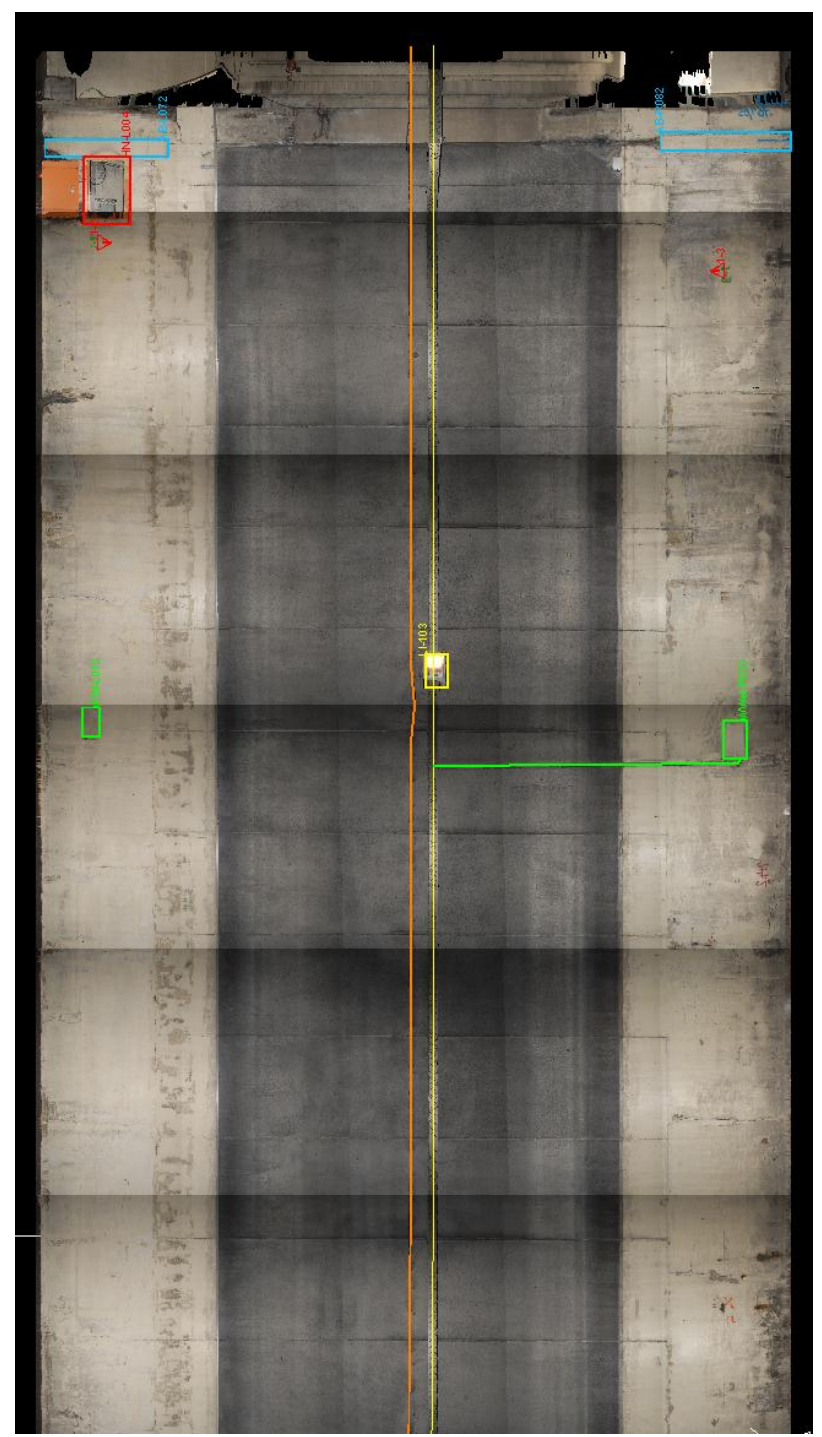

Figure 3. $35 \mathrm{~m}$ tunnel band showing the data of six subsequent scan stations in the onboard control software

\subsection{System test}

To test the system hardware the system has been assembled and calibrated on a test stand in the laboratory, then disassembled and taken to a tunnel site, assembled again and used to measure a $700 \mathrm{~m}$ tunnel section with two different scanner types. Afterwards it has been transported back to lab where the calibration has been repeated. The test showed that the system already performs as desired with regard to measuring and setup time but still needs mechanical improvements to make the platform more robust and the mounting calibration enough stable.

In a further test its suitability for measuring tunnel wall displacements was investigated. Therefore, subsequent wall scans in a tunnel under construction have been carried out, the 3-d displacements processed by a new method (described in the following chapter) and compared to results of conventional surveying. The test revealed a good match of the results.

\section{A METHOD FOR THE EVALUATION OF 3-D TUNNEL WALL DISPLACEMENTS}

In order to provide a spatially well distributed field of displacement vectors, a concept of a regular distribution has been developed. Imagine a developable surface, like a perfect cylinder. Defining a regular grid on its surface leads to an optimal spatial distribution of surface elements. By varying the width of the mesh, the density of the distribution can be adjusted according to the requirements. This notation can be adopted for tunnels, as at least in small sections a tunnel surface comes close to a developable surface, which is sufficient for finding (in consecutive epochs) corresponding surface patches which can be compared through a deformation analysis.

\subsection{Surface Matching by ICP}

If one compares a time series of cylinders, the time-dependent deformation of the surface can be approximated by discretization, i.e. by observing the three-dimensional deviations on the spatial grid points. The practical approach is based on the comparison of differential surface patches around the individual grid points. The minimum extension of the patches depends mainly on the surface topography, which is again dependent on the spatial resolution of the data acquisition, for instance, by terrestrial laserscanning. In order to be able to compare surface patches, on the one hand their topographic characteristics have to be unique, and on the other hand they have to contain shapes which allow unique and accurate matching in all directions. Repeating patterns of topography or very smooth surfaces will lead to ambiguous and wrong or inaccurate results or even failing procedures.

After spreading a regular grid onto the surface of the reference cylinder the surface patches for further processing are defined. Their actual surface is given by the points from the respective laserscanning point cloud. Figure 4 shows the patches which later on will be used in a surface matching process. Here the patches form a gapless pattern of tiles, but they also may be smaller with gaps in between, or they may even overlap. However, the procedure will remain the same.

For matching 3D surface patches the Iterative Closest Point algorithm (ICP) has been applied (Besl and McKay, 1992; Chen and Medioni, 1992), which has been slightly adapted in order to fulfil the requirements of accuracy and speed. The iterative process needs a provisional starting position from which the final optimum surface fit can be approached step by step by applying a spatial orthogonal transformation. Since the deformations from one epoch to the other are small, finding suitable provisional values is not a problem. As result of the ICP the parameters of the transformation are obtained. For each patch seven unknowns are determined, i.e. a scale factor, an orthogonal rotation matrix, which represents the three components of spatial rotation, and the three components of the spatial translation. In the current practical case a scale variation and rotations between two measurements can be neglected, leading to a unit scale and an identity rotation matrix, thus only the translations are of further interest. It is assumed that the transformation parameters refer to the centre of gravity of the respective patch. Eventually, for a better interpretation the transformation parameters are transformed into two tangential components (along and across the axis of the tunnel) and into one radial component (orthogonal to the tunnel surface). 


\subsection{Quality Check}

Although the ICP algorithm is based on a least squares adjustment, the obtained accuracies cannot immediately be applied to the final spatial deformation vectors and also the convergence behaviour does not necessarily indicate the quality and reliability of the actual deformation vectors. Beside the case where the procedure does not converge at all, there are several other reasons why additional quality tests had to be pursued:

- In exceptional cases a significant rotation within one patch might have occurred between two epochs

- There might have been a significant deformation within a patch between two epochs, leading to a conspicuous great $\sigma_{0}$ after the least squares adjustment.

- The result depends highly on the provisional start position, for instance in case of repeating topographical micro-structure where the process may converge to a wrong optimum position.

A series of three empirical tests are carried out for obtaining information about the actually achieved quality:

- After matching seemed to be successful, a full 6 parameter transformation will commence. The translations have already been found by the step before, and therefore all approximate provisional values for translation and rotation can be set to 0 . If after applying this check match, the determined rotation angle remains within a small threshold, the patch is classified as correctly matched.

- $\sigma_{0}$ after ICP is checked against a threshold $\sigma_{0 \max }$. Choosing this threshold depends on the quality of the data, in general on the measurement accuracy of the laserscanner. A reasonable value can be found by analysing all $\sigma_{0}$ values of the project.

- By varying the provisional values (e.g. four times) before commencing the ICP, the convergence behaviour can be checked. In the ideal case, all four processes should deliver the same displacement vectors proving that the matching process was independent of a certain start position and that the correct position was found. Different vectors would indicate unreliable results.

Figure 5 shows the results of the displacement test. On each patch the resulting displacement vector has been drawn in the centre (in green colour), while the results of the four other different start positions appear in other colours. In most cases the matches were successful, one patch in the centre of the second top row shows significantly different displacements and as a consequence, the result needs to be rejected.

In order to facilitate a first quality check, each patch is assigned a colour-coded marker. A red cross means unsuccessful match, either due to insufficient data (as in Figure 5) or due to a diverging matching process. An orange cross stands for significant, but unexpected rotations between consecutive epochs or for a significantly large $\sigma_{0}$. A yellow marker indicates ambiguous convergence behaviour, i.e. a high dependence on provisional values. And eventually a green displacement vector is plotted, if all quality tests have been passed successfully.

It must be mentioned here, that each patch is processed individually. The vectors are not checked whether they are consistent with the overall deformation behaviour or at least with the vectors of the neighbouring patches and whether the entire vector field can be explained physically. Nevertheless, the results and the colour-coded quality measure provide an excellent basis for visual inspection and for a quick detection of anomalies. Figure 6 depicts a section of a check plot. The green vectors show that for most patches in the centre the displacements could be determined reliably, besides, the vector field looks consistent and only along the boundary of the investigated area patches with various kinds of errors have been found.

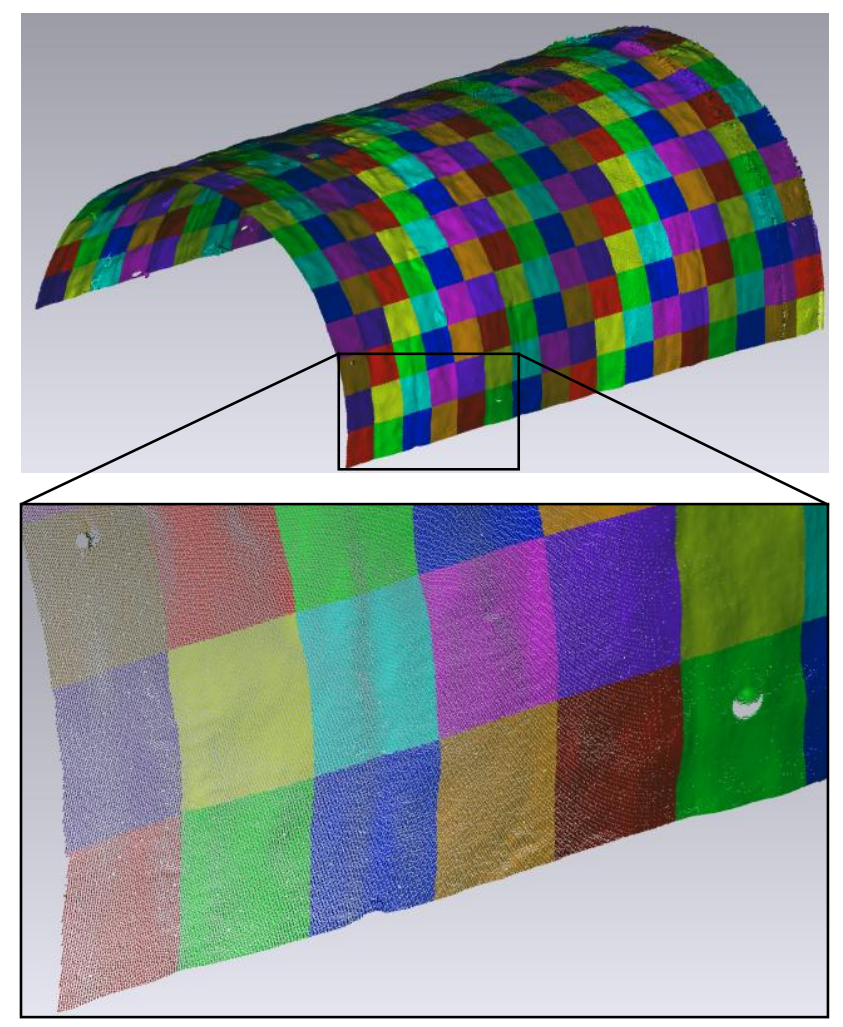

Figure 4. Separation of wall patches from the point cloud.

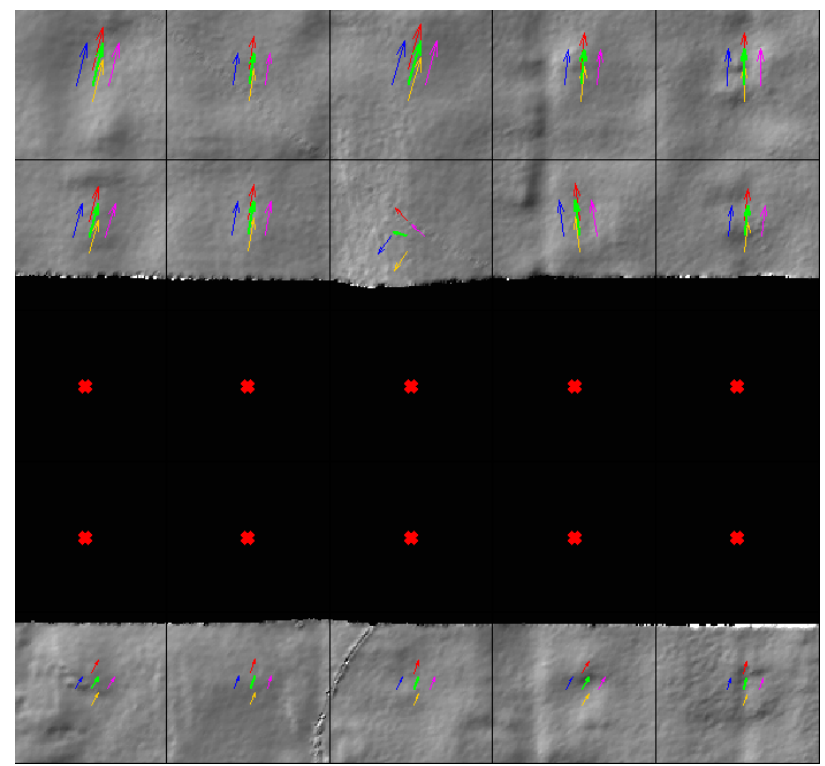

Figure 5. Different displacement vectors resulting from different start values of the ICP-algorithm. 


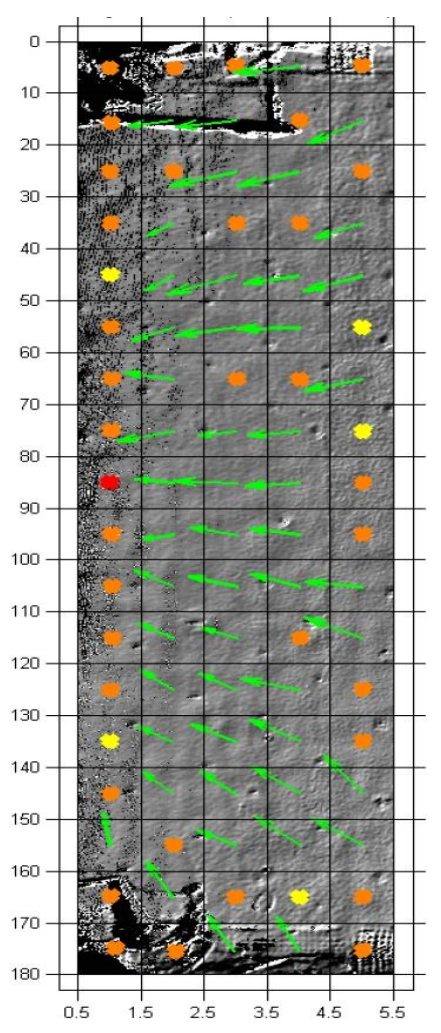

Figure 6. Resulting displacement vectors (tangential component) of all $1 \mathrm{~m}$ patches of a $5 \mathrm{~m}$ tunnel section. The colour-coded crosses indicate matching problems.

\section{A VIRTUAL REALITY TOOL FOR THE SUPPORT OF DATA INTERPRETATION}

To allow for viewing the obtained 3-d displacements in a visual and interactive way and in combination with other geotechnical data a Virtual Reality tool has been developed. By use of this tool the progress of tunnel construction and a variety of measurements can be experienced as real-time 3 -d visualisation. The central part is the reconstruction of the tunnel itself. Engineers can navigate through time and space in an intuitive way and watch how critical measurements change over time. Visualisations appear where the corresponding data is measured and thus its geographic relation is always clear.

The VR tool is a vital support for analysis and interpretation of data. The interface allows switching on and off visualisation classes so that users can focus on data currently relevant for an analysis.

As an example can be represented rod extensometers (Fig. 7) that are installed in boreholes to determine deformations in the geological layers around the tunnel. Typically there are installed several of such boreholes in one measuring cross section, each borehole containing one or more extensometer rods of different length. They extend from a particular position of the tunnel hull into a particular direction, penetrating the surrounding rock or soil. In the VR tool they appear on their correct spatial position. The user interface allows displaying or hiding groups of extensometers that are combined to measure deformations around a tunnel profile.

Investigations on some visualisation metaphors resulted in the usage of flags to indicate extensometer measuring values. The length of these flags change over time according to the timeseries of measurements. They appear at the end of each segment and two more flags below show the last two measurements to provide a short data history. To distinguish between expansion and contraction on a first glance, red and blue flags are shown respectively and placed to the right or left side of the extensometer segment. When changing the viewpoint the flags turn around their extensometer segments so that they face the user and the meaning of their left/right placement is preserved.

When clicking on the 3 -d model of an extensometer, numerical values appear beside flags so that engineers can read the accurate values. Another click hides this text display again. As time progresses and measurements get outdated flags become transparent.

Preliminary user studies show that this kind of visualisation provides a quick comprehension of multiple extensometer measurements. Engineers can zoom into a particular 3-d model of an extensometer and get concrete values with a simple click. Going back and forth in time displays the data history and allows surveying the development of rock/soil deformations in a visual way and in real-time. Figure 7 shows a screenshot of the VR tool with extensometers.

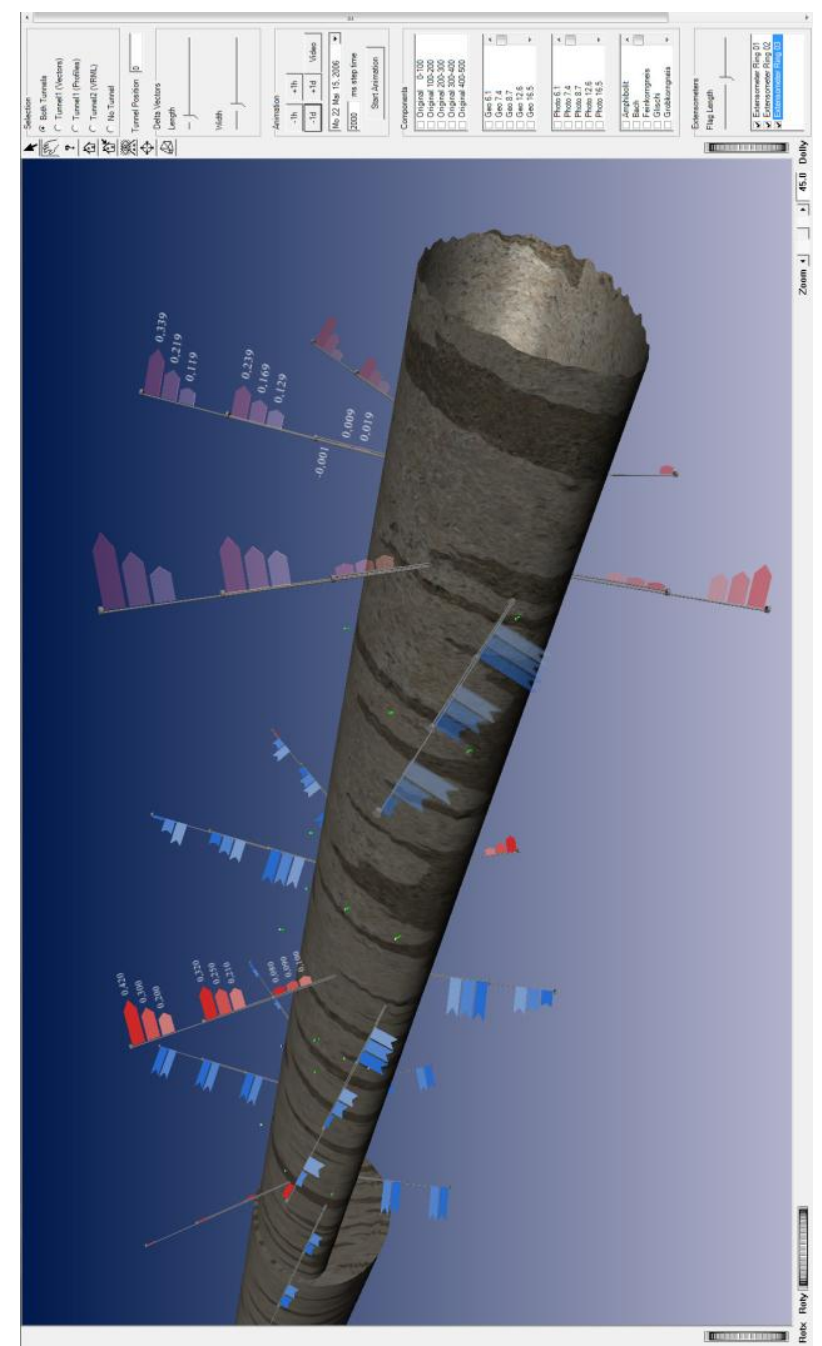

Figure 7: Virtual Reality tool showing the tunnel and three extensometer measuring cross sections. 
To now allow for a combined analysis of extensometer data and tunnel wall displacement data obtained from laserscanning the hull of a reconstructed tunnel model can be viewed with different layer types. Figure 8 shows such a visualisation with extensometers, 3-d tunnel wall displacement vectors and also a colour-coded representation of radial wall displacements.

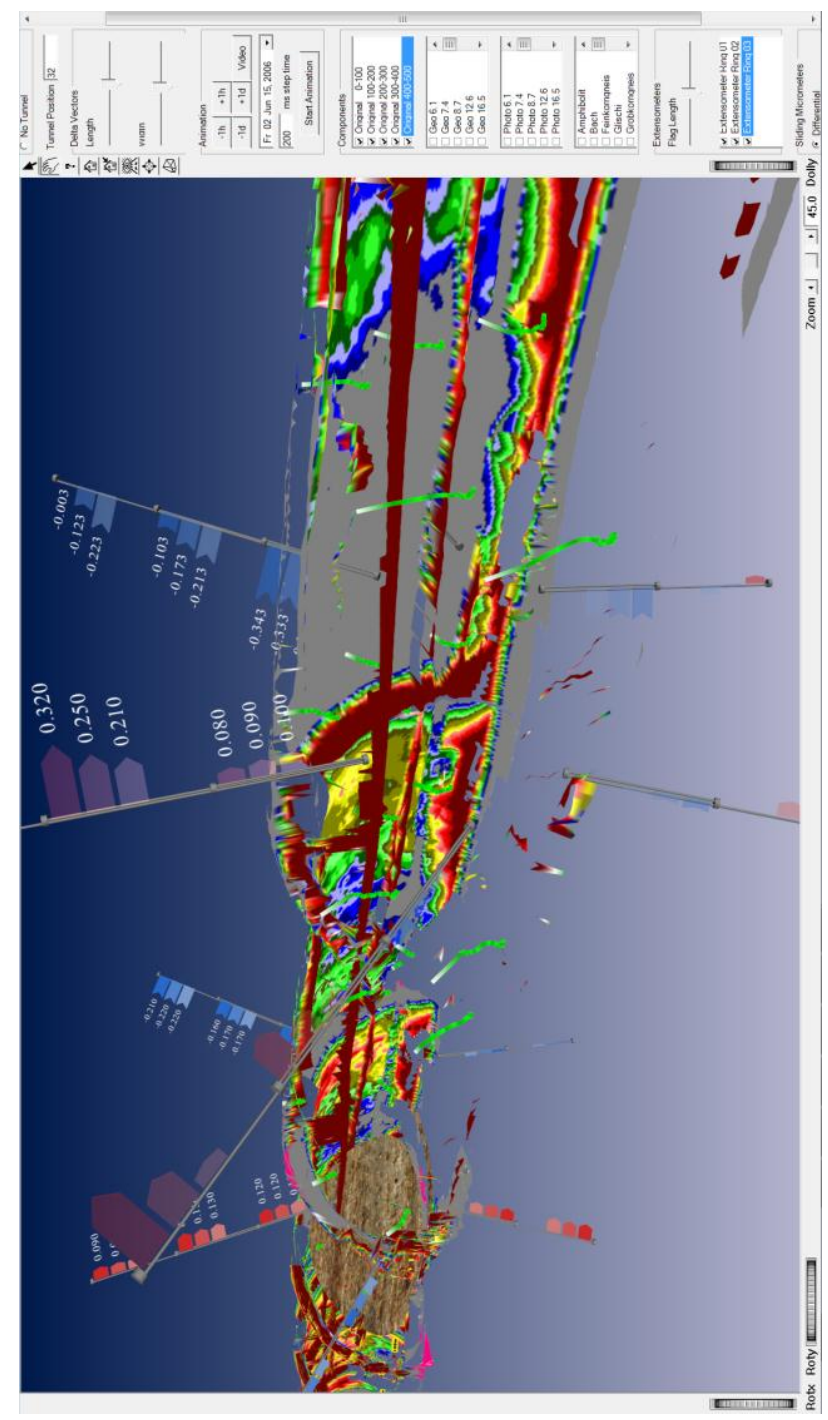

Figure 8: Virtual Reality tool showing tunnel wall displacements obtained from laserscanning and extensometers.

It can be imagined that the interpretation of Figure 8 already heavily challenges even a well-experienced geotechnical expert; even more the VR tool challenges him/her when it then dynamically shows the change of data over time. So for first users, a certain time for training is needed.

\section{CONCLUSION}

The paper reports on three research projects focussing on different aspects of the application of laserscanning for the 3-d displacement monitoring of tunnels under construction. In tunnelling practice, due to several shortcomings, laserscanning will not be able to fully replace the currently executed method in the near future. Although meaningful benefits could be expected and new insights in the deformation behaviour of tunnels gained, a change of paradigm must further stay a dream of geodetic and geotechnical engineers. The technology is already found mature enough with regard to laserscanner hardware and also in scan data processing significant progress has been made and suitable algorithms and methods found. However, the time-is-money aspect is still the limiting factor and so the technology can not yet compete with the well established optical 3-d displacement monitoring method based on totalstation measurements.

However, as both laserscanners and totalstations are used daily during tunnel construction but for different purposes, it is seen a possibility if they are integrated into one system like the presented mobile multi sensor system Orthos Plus. With this system, especially at the critical area close to the tunnel face, the tunnel wall can be mapped and its deformation monitored at least for a few epochs. In this way, laserscanning and conventional surveying with a totalstation will not compete but perfectly supplement each other.

\section{References}

Besl, P.J., McKay, N. (1992): A method for registration of 3-d shapes. IEEE Transactions on Pattern Analysis and Machine Intelligence, 14(2), pp. 239-256.

Chen, Y., Medioni, G. (1992). Object modeling by registration of multiple range images. Image and Vision Computing, 10(3), pp. 145-155.

\section{Acknowledgements}

The described research projects have been supported by the national Austrian research promotion agency FFG and the Austrian Christian Doppler Research Association. 